\title{
Domain of parameters in a model with density- dependent quark mass
}

\author{
Assis, F.S.D.C. ${ }^{1}$ \\ Centro Brasileiro de Pesquisas Físicas \\ Rua Dr. Xavier Sigaud, 150, Urca, Rio de Janeiro, Brazil \\ E-mail: fsdcalibest.com.br
}

\section{Duarte, S.B.}

Centro Brasileiro de Pesquisas Físicas

Rua Dr. Xavier Sigaud, 150, Urca, Rio de Janeiro, Brazil

E-mail: sbdacbpf.br

\begin{abstract}
The strange quark matter in the CFL (Color-Flavor-Locked) superconducting phase at zero temperature has been taken as the ground state of Quantum Chromodynamics (QCD) for light flavors ( $\mathrm{u}, \mathrm{d}, \mathrm{s}$ ) [1]. In this work, the CFL matter is being studied in a phenomenological approach where the interaction between quarks is taken into account by incorporating a density dependence of the in-medium quark mass in the calculation. The pairing effect is considered in the thermodynamical potential of the system, taking the CFL superconducting gap as a free parameter. The stability of the superconducting quark matter is discussed analyzing the equation of state of the system for different values of the parameters used for the description of the employed phenomenological model. The main purpose here is to study the limit values of these parameters to maintain the CFL phase as the lowest energy state of the quark matter.
\end{abstract}

XXXIV edition of the Brazilian Workshop on Nuclear Physics,

Foz de Iguaçu, Paraná state, Brasil

5-10 June 2011

1 Speaker 


\section{Introduction}

Nowadays the main objective of experiments at the high energies regime is focused on demonstrating that the accelerated nucleons can reach energies high enough to dilute its nucleons into a quark and gluon plasma state (QGP). This situation is supported by one fundamental concept in QCD, the asymptotic freedom, which establish that when submitted to extreme density conditions, or very high temperature, quarks behave as free particles. The conversion of quarks confined in the hadrons to plasma state features a typical phase transition. The high density condition can be reached in deep interior of neutron stars, where one believes that the quark-hadron transition may occurs at low temperature. Also there is the possibility that in the primordial universe this quark-hadron phase transition had occurred, a few microseconds after the Big Bang, in an extreme high temperature condition. Within this cosmological scenario, there could happen the formation of dense stable bubbles of strange quark matter with density few times larger than the equilibrium nuclear density, called strangelets. The properties of the strange matter formed have been investigated intensively nowadays, particularly in the CFL phase.

Chakrabarty [2] has used a thermodynamic description of unpaired strange matter, in order to study its properties using the model of density dependent quark mass, which is introduced in such way that, for low densities, quarks become extremely massive and at very high densities the dynamical effective mass vanishes. With this mass dependence the model tries to imitate the regimes of confinement and asymptotic freedom, as we will see latter. The stability in the CFL phase is favored because the pair of quarks is formed in a process similar to that one arising in electric superconductivity forming Cooper pairs. The pairing for quarks of different flavors and colors decreases the energy of the system leading to a more stable QGP condensed state.

In the present study, Chakrabarty model is applied for the superconducting quark matter in CFL-phase to establish the domain of the model parameters where the CFL-phase is taken as the minimum energy state of the system. This domain has already been determined for the unpaired strange matter in Ref. [3] and for the MIT bag model in CFL-phase in Ref. [4]. Here the idea is to determine the parameters for the Chakrabarty density-dependent quark mass model. In the following section the model is presented in more detail, and the main results and conclusions are presented.

\subsection{The Equation of State for Color Superconducting Quark Matter}

In this paper we consider strange matter in at a negligibly low temperature (i.e., zero temperature), so that the gas can be treated as a completely degenerate gas. As a consequence, the strange matter system behaves as a Fermi gas, composed of quarks $u, d$ and s (antiquarks not considered in the limit $\mathrm{T}=0$ ), where the effective masses of quarks is a function of baryonic density $\rho_{B}$ system $[2,3]$, given by

$$
m_{u}=m_{d}=\frac{C}{3 \rho_{B}} \quad m_{s}=m_{s 0}+\frac{C}{3 \rho_{B}}
$$


where $m_{s 0}$ (the current mass of the strange quark) and $\mathrm{C}$ are parameters of the model.

The color superconductor quark matter in the CFL phase has the following characteristics:

i. color gauge and chiral simetries are broken in the barionic regime of density of interest;

ii. the effective quark coupling constant can not be considered weak enough to permits pertubative calculations;

iii. The phase has a neutral eletromagnetic charge in the bulk [1], because in the CFL phase the different quark flavors density are equal, with a commum Fermi momenta to minimizes the free energy of the system.

The effect of quarks pairing in the fermions superconductivity is included in the thermodynamic treatment by means of an additional gap term in the thermodynamic potential of free Fermi gas [5]. Thus, the density of the thermodynamic potential is described as

$$
\Omega=\sum_{i}-\frac{3 m_{i}^{4}}{8 \pi^{2}} f(x)-\frac{3}{\pi^{2}}\left(\mu v^{3}+\Delta_{C F L}^{2} \mu^{2}\right)
$$

where $m_{i}$ are the masses of quarks with different flavors i, $\mu$ is the quark chemical potential and $\Delta_{C F L}$ is the gap energy. The commom Fermi momentum $v$ is given by

$$
v=\left[\left(2 \mu-\sqrt{\mu^{2}+\frac{m_{s}^{2}-m_{u}^{2}}{3}}\right)^{2}-m_{u}^{2}\right]^{1 / 2}
$$

The total pressure $\mathrm{P}$ is to describe the dynamic confinement and asymptotic freedom. For this phase the pressure is defined by

$$
P=-\left.\frac{\partial \Omega}{\partial V}\right|_{T=0, \mu}=-\left.\frac{\partial\left(\Omega / \rho_{B}\right)}{\partial\left(1 / \rho_{B}\right)}\right|_{T=0, \mu}=\left.\rho_{B} \frac{\partial \Omega}{\partial \rho_{B}}\right|_{T=0, \mu}-\Omega=B^{*}-\Omega .
$$

The additional term $\mathrm{B}^{*}$ arises due to the dependence of the effective mass on the baryonic density, and it also plays the role of confining pressure exerted by the vacuum (as in the MIT bag model), we get a situation of confinement in this model [6]. We find, therefore

$$
P=\sum_{i} \frac{3 m_{i}^{4}}{8 \pi^{2}}\left[f(x)+\frac{4}{m_{i}} \frac{C}{3 \rho_{B}} g(x)\right]+\frac{3}{\pi^{2}}\left(\mu v^{3}+\Delta_{C F L}^{2} \mu^{2}\right)
$$

and

$$
B^{*}=\sum_{i} \frac{m_{i}^{3} C g(x)}{2 \pi^{2} \rho_{B}}
$$

With the auxiliary functions $\mathrm{f}(\mathrm{x})$ and $\mathrm{g}(\mathrm{x})$, given by

$$
\begin{gathered}
f(x)=\ln \left(x+\sqrt{x^{2}+1}\right)-x\left(2 x^{2}+1\right) \sqrt{x^{2}+1} \\
g(x)=\ln \left(x+\sqrt{x^{2}+1}\right)-x \sqrt{x^{2}+1}
\end{gathered}
$$


where

$$
x=\frac{v}{m_{i}} .
$$

The particle density for quark $\mathrm{i}$, is defined as

$$
\rho_{i}=-\left.\frac{\partial \Omega}{\partial \mu}\right|_{T=0, \rho_{B}}=\frac{3}{\pi^{2}}\left(v^{3}+2 \Delta_{C F L}^{2} \mu\right) .
$$

Since the CFL phase has the same density of quarks with distinct flavors, the baryon number can be written as follows

$$
\rho_{B}=\frac{\rho_{i}}{3}=\frac{v^{3}}{\pi^{2}}+\frac{2 \Delta_{C F L}^{2} \mu}{\pi^{2}}
$$

The equation of state for cold strange matter in a color superconducting phase is obtained from the known thermodynamic relation

$\varepsilon=-P+\sum_{i} \rho_{i} \mu_{i}=-P+3 \rho_{B} \mu=\sum_{i}-\frac{3 m_{i}^{4}}{8 \pi^{2}}\left[f(x)+\frac{4}{m_{i}} \frac{C}{3 \rho_{B}} g(x)\right]+\frac{3 \Delta_{C F L}^{2} \mu^{2}}{\pi^{2}}$.

The energy per particle as a function of baryon number density is given by Fig. 1 for three different values of the gap, keeping constant the model parameters $\left(\mathrm{C}=70 \mathrm{MeV} \mathrm{fm}{ }^{-3} \mathrm{e} \mathrm{m}_{\mathrm{s} 0}=\right.$ $150 \mathrm{MeV}$ ).

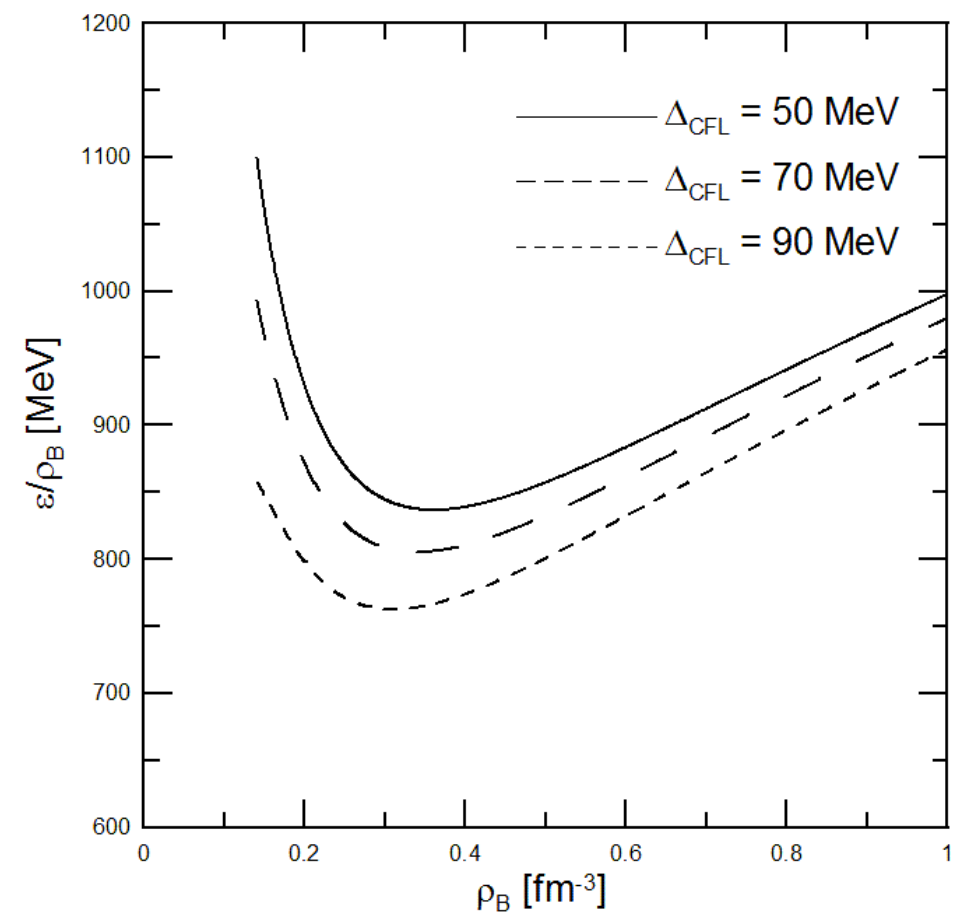

Fig. 1 - Energy per baryon as a function of the baryon density at zero temperature for different value of the gap. 


\section{Results and Conclusions}

The question of stability in a strange matter system depends on the values assumed by the parameters that characterize the properties of the interacting particles and on the intensity of the interaction $[7,8]$. The high stability of the ${ }^{56} \mathrm{Fe}$ nucleus (the binding energy per nucleon is maximum at $\mathrm{A} \approx 56$ ) requires that to have the strange quark matter as the ground state of the quark matter, the energy per unit of baryonic number should satisfies the condition $\left(\varepsilon / \rho_{\mathrm{B}}\right)_{\mathrm{P}=0} \leq 930 \mathrm{MeV}[9-10]$.

In Fig. 2 we show the domain of model parameters for the CFL stability. The lowest limit of $\mathrm{C}$ parameter is around $70 \mathrm{MeV}$, and is fixed by the condition of having quarks confined to non strange hadrons at nuclear density in equilibrium with $\Delta_{\mathrm{CFL}}=0 \mathrm{MeV}$.

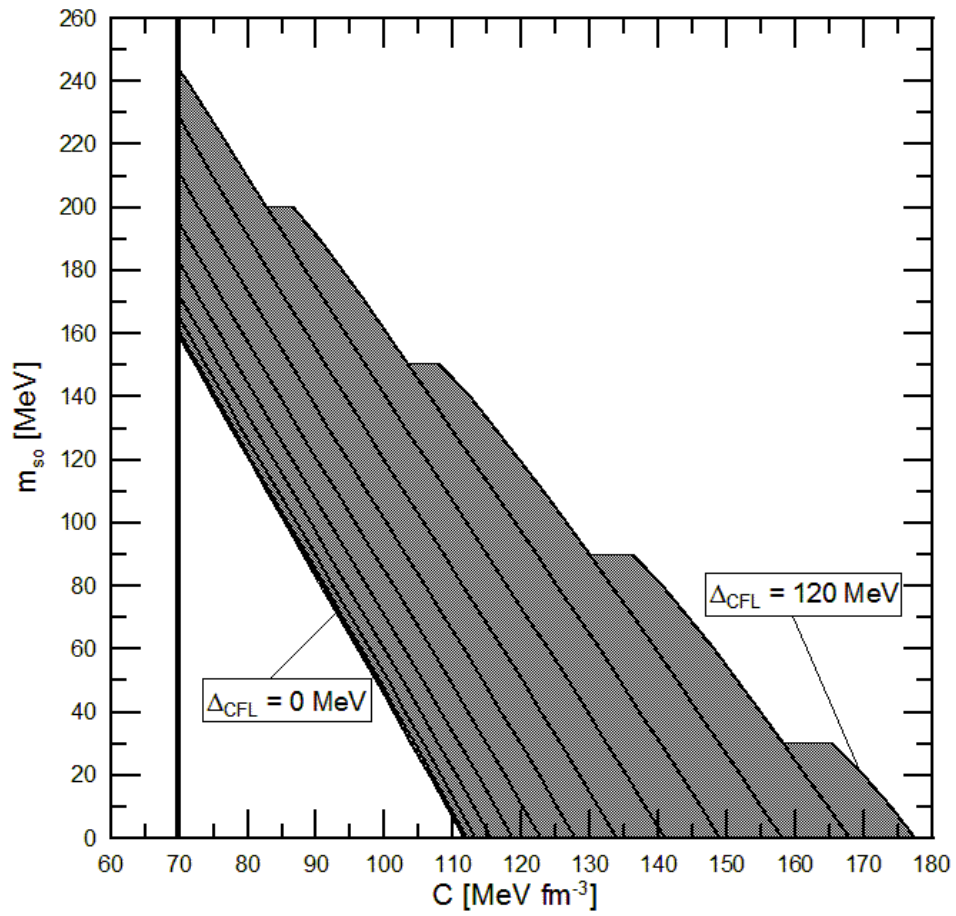

Fig. 2 - Stability window of the parameters in the density-dependent quark mass model for CFL phase with gap $\left(\Delta_{\mathrm{CFL}}\right)$ values ranging from 0 to 120 , in steps of $10 \mathrm{MeV}$. The continuous curves mark the limits where the CFL phase is more stable than the hadronic matter in ${ }^{56} \mathrm{Fe}$.

It's important to stress that (as occurs for the unpaired phase), the situation of zero pressure does not correspond to a minimum of energy per baryon (solid points in Fig. 1). This is a consequence of the extra term, $\mathrm{B}^{*}$, which appears in the pressure for the thermodynamic consistence of the Eq. [1]. Also we note that as the parameter $\Delta_{\mathrm{CFL}}$ gap increases, the CFL phase becomes more stable (as shown in Fig. 1).

The calculated energy density together with the condition $\left(\varepsilon / \rho_{\mathrm{B}}\right)_{\mathrm{P}=0} \leq 930 \mathrm{MeV}$ determines the model parameters domain $\left(\mathrm{C}, \mathrm{m}_{\mathrm{s} 0}\right.$ e $\left.\Delta_{\mathrm{CFL}}\right)$. This result is of fundamental 
importance to discuss use the model to describe the quark-hadron phase transition when establishing hybrid neutron star configuration, which is a work in progress.

\section{References}

[1] Rajagopal, K., \& Wilczek, F. 2001a, Phys. Rev. Lett., 86, 3492.

[2] Chakrabarty, S. Raha, B. Sinha, Phys. Lett. B 229, 112.

[3] O. G. Benvenuto and G. Lugones, Phys. Rev. D51, 1989 (1995)

[4] G. Lugones and J. E. Hovarth, A\&, 4031 (2003) 173-178

[5] H. Rodrigues, S. B. Duarte, J. C. T de Oliveira, The Astrophysical Journal 730:31 (9pp), 2011.

[6] M. Orsaria, H. Rodrigues, S. B. Duarte, Braz. J. Phys. 35, 858 (2005).

[7] E. Witten, Phys. Rev. D 30, 272 (1984).

[8] M. Orsaria, H. Rodrigues, S. B. Duarte, Int. J. Mod. Phys. D16, 291 (2007).

[9] P. Wang, Phys. Rev. C62, 015204.

[10] W. M. Alberico, A. Drago, C. Ratti, Nuclear Physics A 706 (2002). 